\section{Prohexadione-calcium Suppression of Apple Tree Shoot Growth as Affected by Spray Additives}

\author{
R.E. Byers ${ }^{1}$, D.H. Carbaugh ${ }^{2}$, and L.D. Combs ${ }^{2}$ \\ Virginia Polytechnic Institute and State University, Alson H. Smith, Jr. \\ Agricultural Research and Extension Center, 595 Laurel Grove Road, \\ Winchester, VA 22602
}

Additional index words. ethephon, $\mathrm{pH}$, adjuvants, fruit cracking, return bloom, plant growth regulators

\begin{abstract}
Technical grade prohexadione-calcium (93.2\% a.i. P-Ca) applied to 'Fuji'/M.9 trees in three applications in deionized water reduced shoot growth by $25 \%$, but the addition of $\left(\mathrm{NH}_{4}\right)_{2} \mathrm{SO}_{4}$ to $\mathrm{P}$-Ca suppressed shoot growth by $47 \%$. If $\mathrm{P}$-Ca was mixed in well water (high in calcium salts), P-Ca did not suppress shoot growth at all. The commercially formulated prohexadione-calcium [Apogee: $27.5 \% \mathrm{P}-\mathrm{Ca}+\mathbf{5 6 . 1 \%}\left(\mathrm{NH}_{4}\right)_{2} \mathrm{SO}_{4}+\mathbf{1 6 . 4 \%}$ other proprietary additives] + Regulaid in well water (high calcium) was not as effective (reduced growth by $30 \%$ ) as when additional $\left(\mathrm{NH}_{4}\right)_{2} \mathrm{SO}_{4}$ was added (reduced growth by $53 \%$ ), and if $\mathrm{CaCl}_{2}$ (used to control corking) was tank mixed with Apogee + Regulaid, the $\mathrm{Ca}^{++}$interfered with the growth suppression of $\mathrm{P}$-Ca. If $\left(\mathrm{NH}_{4}\right)_{2} \mathrm{SO}_{4}$ was added at the same rate as $\mathrm{CaCl}_{2}(\mathrm{w} / \mathrm{w})$, the Apogee growth suppression was completely restored (reduced growth by $50 \%$ ). Choice (a commercial water conditioner that has $\left(\mathrm{NH}_{4}\right)_{2} \mathrm{SO}_{4}$ in the formulation, among other ingredients) + Li-700, or $\left(\mathrm{NH}_{4}\right)_{2} \mathrm{SO}_{4}+$ Silwet $\mathrm{L}-77$, or $\left(\mathrm{NH}_{4}\right)_{2} \mathrm{SO}_{4}$ + Silwet L-77 + Oil were among the most effective adjuvant combinations with Apogee. The addition of ethephon at $270 \mathrm{mg} \cdot \mathrm{L}^{-1}$ improved the growth suppression of Apogee + $\left(\mathrm{NH}_{4}\right)_{2} \mathrm{SO}_{4}+$ Regulaid. Solubor compromised the effectiveness of Apogee + Regulaid. Adjusting the $\mathrm{pH}$ of the Apogee $+\left(\mathrm{NH}_{4}\right)_{2} \mathrm{SO}_{4}+$ Regulaid spray to either $\mathrm{pH}=4$ or $\mathrm{pH}$ $=9$ did not affect efficacy. The combination of Apogee $+\left(\mathrm{NH}_{4}\right)_{2} \mathrm{SO}_{4}+$ Regulaid caused increased fruit cracking of 'Empire' fruit as compared to the control (7\%), presumably due to increased absorption of P-Ca. Chemical names used: Prohexadione-calcium (P-Ca, 3-oxido-4-propionyl-5-oxo-3cyclohexenecarboxylate) formulated as BAS-125 (10\% P-Ca); Apogee (27.5\% P-Ca), or Technical 93.5\% P-Ca); Regulaid (polyoxyethylenepolypropoxypropanol, alkyl 2-ethoxethanol, and dihydroxy propane); Silwet L-77 (polyalkyleneoxide modified heptametyltrisiloxane, silicon surfactant), LI-700 (80\%, phosphatidylcholine, methylacetic acid and alkyl polyoxyethylene ether); Superior Oil (Drexel Damoil 70-second delayed dormant spray oil); ethephon (2-chloroethyl phosphonic acid); Solubor $(20.5 \%$, Boron equivalent); captan ( $N$-Trichloromethylthio-4-cyclohenene-1,2-dicarboximide).
\end{abstract}

Several plant growth regulators have been used to reduce vegetative growth of fruit trees, thereby reducing pruning costs and improving fruit quality (Faust, 1984; Looney, 1983; Luckwill,1970; Miller, 1988; Williams, 1984). Apogee $\left[\left(27.5 \% \mathrm{P}-\mathrm{Ca}+72.5 \% \mathrm{NH}_{4} \mathrm{SO}_{4}\right)\right.$ BASF Corp., Research Triangle Park, N.C.], recently registered as a foliar aqueous spray for inhibiting shoot growth of apple [Malus $\times$ sylvestris (L.) var. domestica (Borkh. Mansf.] trees, results in reduced pruning time, tree canopy size and density, and reduced severity of fireblight (Byers and Yoder, 1999; Yoder et al., 1999).

Since prohexadione-calcium (P-Ca) sprays are rather expensive, it is important to maximize the efficacy of the applied dose by promoting absorption, translocation, or both, to the site of GA synthesis (Rademacher, 2000). The reduced effectiveness of many pesticides have been related to chemical stability in aqueous

Received for publication 13 Mar. 2002. Accepted for publication 14 Feb. 2003. Appreciation to Seth Combs, Heath Combs, Jean Engleman, Maurice Keeler, and Harriet Keeler for data collection, analysis, and technical assistance.

${ }^{1}$ Professor.

${ }^{2}$ Research Specialist. spray solutions at high or low $\mathrm{pH}$, the sprayer water volume applied per hectare, physical barriers related to the plant cuticle structure, calcium salts in the spray water (Nalewaja et al., 1992), calcium or boron nutrients routinely added to sprays to correct physiological disorders of apple (Raese and Drake, 2002), photooxidation at high light intensities (Rademacher unpublished), energetics and/or mechanics of plant growth regulator loading into the phloem or xylem cells, binding or metabolism of the chemical before it reaches the site of action or some other action. Many adjuvants, alone or incombination with other chemicals, added to sprays may potentiate or interfere with the activity of specific PGRs, but frequently their modes of action are not understood.

The objectives of the experiments reported here were to evaluate the effectiveness of $\mathrm{P}$ $\mathrm{Ca}$ [formulated as the BAS-125 10\% P-Ca), Apogee (27.5\% P-Ca), or the technical (93.2\% $\mathrm{P}-\mathrm{Ca})$ ], alone or in combinations with deionized water, well water (high in calcium), calcium chloride, solubor, ammonium sulfate, commercial spray-adjuvants, $\mathrm{pH}$ of spray water, and ethephon for influences on tree-growth and subsequent flowering.
General. Randomized complete-block designs were used in all experiments. Trees were blocked by location within rows or by trunk cross-sectional area (TCSA). All experiments had six blocks or more. Trees were sprayed with a low pressure hand-wand sprayer to drip. In the dormant season (Expts. 1-4), average shoot length of the longest top two shoots, length of 4 or 10 longest scaffold shoots, weight, and basal and terminal shoot diameters of these scaffold shoots, total length of all shoots longer than 30 $\mathrm{cm}$, time required to prune each tree, number of cuts/tree, and pruning weights $/ \mathrm{cm}^{2}$ TCSA per tree were recorded. Data were analyzed by analysis of variance (ANOVA) and General Linear Method (GLM) procedures using SAS software (SAS Institute, 1985). Means were compared by single-degree-of-freedom contrasts, by linear and polynomial regressions, or Duncan's New Multiple Range Test depending upon the experimental design.

Expt. 1. In 1999, 102 five-year-old 'Fuji'/ M.9 trees (six blocks) were selected for 17 treatments (Table 1). Apogee was applied at petal fall $(\mathrm{PF}), \mathrm{PF}+21 \mathrm{~d}$, and $\mathrm{PF}+42 \mathrm{~d}$ to the same trees at $125 \mathrm{mg} \cdot \mathrm{L}^{-1}$ either alone or in combination with $\mathrm{CaCl}_{2}$, adjuvants [Regulaid (KaloAgricultural Chemicals, Overland Park, Kans.), Silwet L-77 (OSI Industries, Tarrytown, N.Y.), Superior Oil (Drexel Chemical Co., Memphis, Tenn.)], hard water, or ammonium sulfate. In addition, two P-Ca formulations (Apogee and BAS 125) were evaluated. Shoot length of the four longest scaffold shoots were measured on 15 June 1999. In the dormant season, the total length of shoots longer than $30 \mathrm{~cm}$, weight and time required to prune trees, number of cuts, and pruning weights $/ \mathrm{cm}^{2}$ TCSA were taken.

Expt. 2. In 2000, 147 six-year-old 'Fuji'/ M.9 trees (seven blocks) were selected for 21 treatments (Table 2). Apogee was applied at 125 $\mathrm{mg} \cdot \mathrm{L}^{-1}$ in three applications to the same trees at $\mathrm{FB}+4, \mathrm{FB}+21$, and $\mathrm{FB}+42 \mathrm{~d}$. In addition, ethephon, Regulaid, $\left(\mathrm{NH}_{4}\right)_{2} \mathrm{SO}_{4}$, Superior Oil, Choice (Loveland Industries, Greeley, Colo), Li-700 (Loveland Industries), $\mathrm{NaCl}, \mathrm{KCl}$, $\mathrm{CaCl}_{2}, \mathrm{NaOH}, \mathrm{HCl}$ and captan were applied in various combinations with Apogee as potential adjuvants, water conditioners, effectors, or to adjust $\mathrm{pH}$ (Table 2). On 26 June, 10 of the longest scaffold shoots were tagged around the periphery of each tree and their lengths were measured. During dormancy, these 10 scaffold shoots were removed and the basal diameters and shoot lengths were recorded. In addition, the nodes $/ \mathrm{cm}$ of the basal $40 \mathrm{~cm}$ and nodes $/ \mathrm{cm}$ of the upper $30 \mathrm{~cm}$ of shoots were recorded. Trees were pruned and the total length of the shoots longer than $30 \mathrm{~cm}$, the weight and time required to prune, the number of cuts, and the pruning weights $/ \mathrm{cm}^{2}$ TCSA per tree were recorded.

Expt.3.In 2001,78 six-year-old 'Fuji'/M.9 trees (six blocks) were selected for 13 treatments (Table 3). P-Ca formulations [Apogee $27.5 \%$ a.i. P-Ca) or technical pro-hexadionecalcium (93.2\% a.i. P-CA)] were applied at $125 \mathrm{mg} \cdot \mathrm{L}^{-1} \mathrm{P}-\mathrm{Ca}$ in three applications to the same trees at $\mathrm{FB}+7, \mathrm{FB}+28$, and $\mathrm{FB}+59$ 


\section{Growth Regulators}

Table 1. Effect of P-Ca, adjuvants, and nutrient sprays on shoot growth of 'Fuji'/M.9 applied in 1999.

\begin{tabular}{|c|c|c|c|c|c|c|c|c|c|c|c|}
\hline \multirow[b]{3}{*}{ Treatment $^{\mathrm{y}}$} & \multirow{3}{*}{$\begin{array}{c}\mathrm{P}-\mathrm{Ca} \\
\text { formulation } \\
\left(\mathrm{mg} \cdot \mathrm{L}^{-1}\right)\end{array}$} & & & & & & \multirow{3}{*}{$\begin{array}{c}\text { June } 1999 \\
\text { Shoot } \\
\text { length }(\mathrm{cm}) \\
\text { scaffold }^{x}\end{array}$} & \multicolumn{4}{|c|}{ Dec. 1999} \\
\hline & & \multicolumn{5}{|c|}{ Added chemicals } & & \multirow{2}{*}{$\begin{array}{c}\text { Shoot } \\
\text { length }(\mathrm{cm}) \\
\text { scaffold }^{\mathrm{w}}\end{array}$} & \multirow{2}{*}{$\begin{array}{c}\text { Shoot } \\
\text { wt (g) } \\
\text { scaffold }^{\text {w }}\end{array}$} & \multirow{2}{*}{$\begin{array}{l}\text { Basal } \\
\text { diam } \\
(\mathrm{cm})\end{array}$} & \multirow[b]{2}{*}{ Cuts/tree } \\
\hline & & Regulaid & Oil & Silwet L-77 & $\mathrm{CaCl}_{2}$ & $\left(\mathrm{NH}_{4}\right)_{2} \mathrm{SO}_{4}$ & & & & & \\
\hline 1. & No treatment & - & -- & - & - & - & $51.7 \mathrm{a}$ & $53.0 \mathrm{a}$ & $10.6 \mathrm{a}$ & $0.712 \mathrm{a}$ & $75.6 \mathrm{a}$ \\
\hline 2. & BAS $125(10 \%)$ & + & - & - & - & - & $36.2 \mathrm{c}$ & $37.1 \mathrm{ef}$ & $7.3 \mathrm{~b}-\mathrm{d}$ & $0.672 \mathrm{a}-\mathrm{d}$ & $64.1 \mathrm{ab}$ \\
\hline 3. & BAS $125(10 \%)$ & - & + & + & - & - & $32.6 \mathrm{c}$ & $33.4 \mathrm{f}$ & $5.8 \mathrm{~d}$ & $0.573 \mathrm{de}$ & $63.1 \mathrm{ab}$ \\
\hline 4. & Apogee $(27.5 \%)$ & - & - & - & -- & - & $43.6 \mathrm{~b}$ & $46.7 \mathrm{bc}$ & $9.4 \mathrm{a}-\mathrm{c}$ & $0.669 \mathrm{a}-\mathrm{d}$ & $66.1 \mathrm{ab}$ \\
\hline 5. & Apogee $(27.5 \%)$ & + & - & - & - & - & $36.3 \mathrm{c}$ & $36.9 \mathrm{ef}$ & $6.4 \mathrm{~cd}$ & $0.604 \mathrm{a}-\mathrm{e}$ & $62.7 \mathrm{ab}$ \\
\hline 6. & Apogee $(27.5 \%)$ & - & + & + & - & - & $34.5 \mathrm{c}$ & $34.1 \mathrm{f}$ & $5.7 \mathrm{~d}$ & $0.557 \mathrm{e}$ & $60.1 \mathrm{~b}$ \\
\hline 7. & Apogee $(27.5 \%)$ & - & - & - & + & - & $46.9 \mathrm{ab}$ & $49.7 \mathrm{a}-\mathrm{c}$ & $9.2 \mathrm{a}-\mathrm{c}$ & $0.696 \mathrm{ab}$ & $67.6 \mathrm{ab}$ \\
\hline 8. & Apogee $(27.5 \%)$ & - & - & - & - & + & $32.1 \mathrm{c}$ & $31.4 \mathrm{f}$ & $5.1 \mathrm{~d}$ & $0.586 \mathrm{c}-\mathrm{e}$ & $59.9 \mathrm{~b}$ \\
\hline 9. & Apogee $(27.5 \%)$ & - & - & - & + & + & $35.0 \mathrm{c}$ & $35.6 \mathrm{ef}$ & $6.7 \mathrm{~cd}$ & $0.647 \mathrm{a}-\mathrm{e}$ & $62.0 \mathrm{ab}$ \\
\hline 10. & Apogee $(27.5 \%)$ & + & - & - & + & - & $44.1 \mathrm{~b}$ & $44.9 \mathrm{~cd}$ & $7.5 \mathrm{~b}-\mathrm{d}$ & $0.632 \mathrm{a}-\mathrm{e}$ & $66.0 \mathrm{ab}$ \\
\hline 11. & Apogee $(27.5 \%)$ & + & - & - & - & + & $31.2 \mathrm{c}$ & $32.5 \mathrm{f}$ & $6.6 \mathrm{~cd}$ & $0.654 \mathrm{a}-\mathrm{e}$ & $61.1 \mathrm{~b}$ \\
\hline 12. & Apogee $(27.5 \%)$ & + & - & - & + & + & $36.1 \mathrm{c}$ & $40.3 \mathrm{de}$ & $9.2 \mathrm{a}-\mathrm{c}$ & $0.692 \mathrm{a}-\mathrm{c}$ & $62.3 \mathrm{ab}$ \\
\hline 13. & Apogee $(27.5 \%)$ & - & + & + & + & + & $33.1 \mathrm{c}$ & $32.0 \mathrm{f}$ & $5.4 \mathrm{~d}$ & $0.589 \mathrm{~b}-\mathrm{e}$ & $64.6 \mathrm{ab}$ \\
\hline 14. & Apogee $(27.5 \%)$ & - & + & + & + & - & $43.2 \mathrm{~b}$ & $44.1 \mathrm{~cd}$ & $7.4 \mathrm{~b}-\mathrm{d}$ & $0.607 \mathrm{a}-\mathrm{e}$ & $58.0 \mathrm{~b}$ \\
\hline 15. & Apogee $(27.5 \%)$ & - & + & + & - & + & $31.5 \mathrm{c}$ & $32.2 \mathrm{f}$ & $6.4 \mathrm{~cd}$ & $0.645 \mathrm{a}-\mathrm{e}$ & $61.7 \mathrm{ab}$ \\
\hline 16. & 0 & + & - & - & + & + & $47.1 \mathrm{ab}$ & $51.5 \mathrm{ab}$ & $10.0 \mathrm{ab}$ & $0.680 \mathrm{a}-\mathrm{d}$ & $66.3 \mathrm{ab}$ \\
\hline 17. & 0 & - & + & + & + & + & $47.8 \mathrm{ab}$ & $49.7 \mathrm{a}-\mathrm{c}$ & $9.3 \mathrm{a}-\mathrm{c}$ & $0.710 \mathrm{a}$ & $64.4 \mathrm{ab}$ \\
\hline \multicolumn{2}{|l|}{ Contrasts } & \multicolumn{4}{|c|}{ Comparisons } & & $\operatorname{Pr}>F^{v}$ & $\operatorname{Pr}>\mathrm{F}$ & $\operatorname{Pr}>\mathrm{F}$ & $\operatorname{Pr}>\mathrm{F}$ & $\operatorname{Pr}>\mathrm{F}$ \\
\hline \multicolumn{2}{|l|}{2,3 vs. 5,6} & \multicolumn{5}{|c|}{ BAS $12510 \%$ vs. Apogee $27.5 \%$} & NS & NS & NS & NS & NS \\
\hline \multicolumn{2}{|c|}{$2,5,12$ vs. $3,6,13$} & \multicolumn{5}{|c|}{ Regulaid vs. Oil + L-77 (+Apogee) } & $*$ & $* *$ & $* *$ & $* *$ & NS \\
\hline \multicolumn{2}{|c|}{$4,5,6$ vs. $7,10,14$} & \multicolumn{5}{|c|}{$\mathrm{CaCl}_{2}$ vs. none (+Apogee) } & $* * *$ & $* * *$ & NS & NS & NS \\
\hline \multicolumn{2}{|c|}{$4,5,6$ vs. $8,11,15$} & \multicolumn{5}{|c|}{$\left(\mathrm{NH}_{4}\right)_{2}^{2} \mathrm{SO}_{4}$ vs. none (+Apogee) } & $* * *$ & $* * *$ & NS & NS & NS \\
\hline \multicolumn{2}{|c|}{$7,10,14$ vs. $8,11,15$} & \multicolumn{5}{|c|}{$\mathrm{CaCl}_{2}$ vs. $\left(\mathrm{NH}_{4}\right)_{2} \mathrm{SO}_{4}(+$ Apogee $)$} & $* * *$ & $* * *$ & $* *$ & NS & NS \\
\hline \multicolumn{2}{|c|}{$7,10,14$ vs. $9,12,13$} & \multicolumn{5}{|c|}{$\mathrm{CaCl}_{2}$ vs. $\mathrm{CaCl}_{2}+\left(\mathrm{NH}_{4}\right)_{2} \mathrm{SO}_{4}(+$ Apogee $)$} & $* * *$ & $* * *$ & NS & NS & NS \\
\hline
\end{tabular}

${ }^{2}$ Full bloom occurred 30 Apr. 1999. Treatments were applied at PF, $\mathrm{PF}+21, \mathrm{PF}+42 \mathrm{~d}$ with a low pressure hand-wand sprayer.

${ }^{y}$ Concentrations $\left(\mathrm{mg} \cdot \mathrm{L}^{-1}\right): \mathrm{P}-\mathrm{Ca}=125 \mathrm{mg} \cdot \mathrm{L}^{-1} ; \mathrm{CaCl}_{2}=1.8 \mathrm{~g} \cdot \mathrm{L}^{-1} ;\left(\mathrm{NH}_{4}\right)_{2} \mathrm{SO}_{4}=1.8 \mathrm{~g} \cdot \mathrm{L}^{-1} ;$ Regulaid $=1.257 \mathrm{~mL} \cdot \mathrm{L}^{-1} ; \mathrm{Oil}=2.514 \mathrm{~mL} \cdot \mathrm{L}^{-1} ; \mathrm{Silwet} \mathrm{L}-77=$ $0.314 \mathrm{~mL} \cdot \mathrm{L}^{-1}$

${ }^{x}$ Four longest scaffold shoots (15 June 1999).

${ }^{w}$ Five longest scaffold shoots (16 Dec. 1999).

Ns, ,*******Nonsignificant or significant at $P \leq 0.05,0.01,0.001$, respectively.

Table 2. Effect of P-Ca (three applications) on shoot growth of 'Fuji'/M.9 applied in 2000 and return bloom in $2001 .{ }^{2}$

\begin{tabular}{|c|c|c|c|c|c|c|c|c|c|}
\hline \multirow[b]{2}{*}{ Treatment ${ }^{y}$} & \multirow[b]{2}{*}{$\begin{array}{l}\text { Apogee } \\
(27.5 \% \\
\text { P-Ca) }\end{array}$} & \multirow[b]{2}{*}{$\begin{array}{c}\text { Added } \\
\text { chemicals }\end{array}$} & \multirow[b]{2}{*}{$\begin{array}{l}\text { Special } \\
\text { sprays }\end{array}$} & \multirow[b]{2}{*}{$\begin{array}{c}\mathrm{pH} \text { of } \\
\text { mix }\end{array}$} & \multirow{2}{*}{$\begin{array}{c}\text { June } 2000 \\
\text { Shoot } \\
\text { length }(\mathrm{cm}) \\
\text { scaffold }^{\mathrm{x}}\end{array}$} & \multicolumn{3}{|c|}{ Dec. 2000} & \multirow{2}{*}{$\begin{array}{c}\text { Apr. } 2001 \\
\text { Return bloom } \\
\text { (\% visual spurs } \\
\text { flowering) }\end{array}$} \\
\hline & & & & & & $\begin{array}{l}\begin{array}{l}\text { Length } \\
(\mathrm{cm}) \\
\text { scaffold }^{w}\end{array}\end{array}$ & $\begin{array}{l}\text { Basal } \\
\text { diam } \\
(\mathrm{mm})\end{array}$ & $\begin{array}{c}\mathrm{Cuts} / \mathrm{cm}^{2} \\
/ \text { tree }\end{array}$ & \\
\hline 1. Control & - & --- & & --- & $56.5 \mathrm{ab}^{\mathrm{t}}$ & $76.3 \mathrm{a}$ & $12.0 \mathrm{ab}$ & $82.0 \mathrm{ab}$ & $70.7 \mathrm{bc}$ \\
\hline 2. & + & --- & & 7.9 & $43.5 \mathrm{~cd}$ & $69.8 \mathrm{a}$ & $11.4 \mathrm{ab}$ & $65.3 \mathrm{~b}-\mathrm{d}$ & $70.7 \mathrm{bc}$ \\
\hline 3. & + & $\left(\mathrm{NH}_{4}\right)_{2} \mathrm{SO}_{4}$ & & 7.6 & $34.8 \mathrm{e}-\mathrm{g}$ & $66.3 \mathrm{a}$ & $11.5 \mathrm{ab}$ & $58.4 \mathrm{~cd}$ & $83.6 \mathrm{ab}$ \\
\hline 4. & + & Urea & & 7.8 & $47.6 \mathrm{~cd}$ & $75.8 \mathrm{a}$ & $12.1 \mathrm{ab}$ & $77.9 \mathrm{a}-\mathrm{c}$ & $72.9 \mathrm{bc}$ \\
\hline 5. & + & $\left(\mathrm{NH}_{4}\right)_{2} \mathrm{SO}_{4}+$ Regulaid & & 7.7 & $34.3 \mathrm{e}-\mathrm{g}$ & $64.8 \mathrm{a}$ & $10.8 \mathrm{~b}$ & $57.6 \mathrm{~cd}$ & $83.3 \mathrm{ab}$ \\
\hline 6. & + & $\left(\mathrm{NH}_{4}\right)_{2} \mathrm{SO}_{4}+\mathrm{Oil}$ & & 7.5 & $31.0 \mathrm{gh}$ & $75.4 \mathrm{a}$ & $11.4 \mathrm{ab}$ & $68.9 \mathrm{a}-\mathrm{d}$ & $67.1 \mathrm{bc}$ \\
\hline 7. & + & Choice + LI-700 & & 4.7 & $32.4 \mathrm{f}-\mathrm{h}$ & $74.7 \mathrm{a}$ & $11.4 \mathrm{ab}$ & $60.6 \mathrm{~b}-\mathrm{d}$ & $80.7 \mathrm{ab}$ \\
\hline 8. & + & $\left(\mathrm{NH}_{4}\right)_{2} \mathrm{SO}_{4}+$ Silwet L-77 & & 7.6 & $30.7 \mathrm{gh}$ & $75.0 \mathrm{a}$ & $11.5 \mathrm{ab}$ & $57.0 \mathrm{~cd}$ & $71.4 \mathrm{bc}$ \\
\hline 9. & + & $\mathrm{NaCl}^{4}$ & & 7.6 & $40.3 \mathrm{~d}-\mathrm{f}$ & $66.6 \mathrm{a}$ & $11.0 \mathrm{ab}$ & $59.4 \mathrm{~b}-\mathrm{d}$ & $77.9 \mathrm{a}-\mathrm{c}$ \\
\hline 10. & + & $\mathrm{KCl}$ & & 7.6 & $45.5 \mathrm{~cd}$ & $78.0 \mathrm{a}$ & $12.0 \mathrm{ab}$ & $74.7 \mathrm{a}-\mathrm{c}$ & $73.3 \mathrm{bc}$ \\
\hline 11. & + & $\mathrm{CaCl}_{2}$ & & 7.6 & $50.6 \mathrm{bc}$ & $72.1 \mathrm{a}$ & $11.9 \mathrm{ab}$ & $73.0 \mathrm{a}-\mathrm{d}$ & $73.6 \mathrm{bc}$ \\
\hline 12. & + & $\begin{array}{l}\left(\mathrm{NH}_{4}\right)_{2}^{2} \mathrm{SO}_{4} \\
\quad+\text { Regulaid }+\mathrm{CaCl}_{2} \\
\left(\mathrm{NH}_{4}\right)_{2} \mathrm{SO}_{4}\end{array}$ & $\begin{array}{l}\text { Tank mixed } \\
\left(\text { High rate } \mathrm{NH}_{4} \mathrm{SO}_{4}\right) \\
\text { Tank mixed }\end{array}$ & 7.4 & $34.1 \mathrm{e}-\mathrm{g}$ & $77.9 \mathrm{a}$ & $11.7 \mathrm{ab}$ & $68.9 \mathrm{a}-\mathrm{d}$ & $74.3 \mathrm{a}-\mathrm{c}$ \\
\hline 14 & + & + Regulaid $+\mathrm{CaCl}_{2}$ & (Low rate $\mathrm{NH}_{4} \mathrm{SO}_{4}$ ) & 7.5 & $\begin{array}{l}45.8 \mathrm{~cd} \\
48.9 \mathrm{~b}-\mathrm{d}\end{array}$ & $78.7 \mathrm{a}$ & $11.9 \mathrm{ab}$ & $66.4 \mathrm{a}-\mathrm{d}$ & $73.6 \mathrm{bc}$ \\
\hline $\begin{array}{l}14 . \\
15 .\end{array}$ & $\begin{array}{l}+ \\
+\end{array}$ & $\begin{array}{l}\mathrm{CaCl} \\
+\mathrm{R}_{2} \text { egulaid }\end{array}$ & Post-trt ${ }^{v}$ & $\begin{array}{l}7.5 \\
7.5 \\
8.0\end{array}$ & $\begin{array}{l}48.9 \mathrm{~b}-\mathrm{d} \\
50.4 \mathrm{bc}\end{array}$ & $\begin{array}{l}81.0 \mathrm{a} \\
81.0 \mathrm{a}\end{array}$ & $\begin{array}{l}11.5 \mathrm{ab} \\
12.3 \mathrm{ab}\end{array}$ & $74.8 \mathrm{abc}$ & $\begin{array}{l}01.9 \mathrm{bc} \\
72.9 \mathrm{bc}\end{array}$ \\
\hline 16. & + & $\mathrm{CaCl}_{2}+$ Regulaid & Pre-trt ${ }^{\mathrm{u}}$ & 7.6 & $41.9 \mathrm{c}-\mathrm{e}$ & $71.8 \mathrm{a}$ & $11.2 \mathrm{ab}$ & $58.6 \mathrm{~cd}$ & $79.3 \mathrm{a}-\mathrm{c}$ \\
\hline 17. & + & $\left(\mathrm{NH}_{4}\right)_{2} \mathrm{SO}_{4}+\mathrm{HCl}$ & Acidic $\mathrm{pH}=4.0$ & 3.9 & $30.6 \mathrm{gh}$ & $66.9 \mathrm{a}$ & $11.0 \mathrm{ab}$ & $61.1 \mathrm{~b}-\mathrm{d}$ & $78.6 \mathrm{a}-\mathrm{c}$ \\
\hline 18. & + & $\left(\mathrm{NH}_{4}\right)_{2} \mathrm{SO}_{4}+\mathrm{NaOH}$ & Basic $\mathrm{pH}=9.0$ & 8.9 & $31.1 \mathrm{gh}$ & $74.0 \mathrm{a}$ & $11.5 \mathrm{ab}$ & $61.6 \mathrm{~b}-\mathrm{d}$ & $77.9 \mathrm{a}-\mathrm{c}$ \\
\hline 19. & + & $\left(\mathrm{NH}_{4}\right)_{2} \mathrm{SO}_{+}^{+}$Regulaid + Captan & & 7.6 & $32.8 \mathrm{f}-\mathrm{h}$ & $68.0 \mathrm{a}$ & $11.0 \mathrm{ab}$ & $56.6 \mathrm{~cd}$ & $76.1 \mathrm{a}-\mathrm{c}$ \\
\hline 20. & + & $\left(\mathrm{NH}_{4}\right)_{2} \mathrm{SO}_{4}+$ Regulaid + Ethephon & & 6.3 & $24.6 \mathrm{~h}$ & $67.1 \mathrm{a}$ & $10.9 \mathrm{ab}$ & $50.9 \mathrm{~d}$ & $90.0 \mathrm{a}$ \\
\hline 21. & - & Regulaid $+\mathrm{CaCl}_{2}+\left(\mathrm{NH}_{4}\right)_{2} \mathrm{SO}_{4}$ & & 7.4 & $63.4 \mathrm{a}$ & $82.2 \mathrm{a}$ & $12.7 \mathrm{a}$ & $87.9 \mathrm{a}$ & $63.6 \mathrm{c}$ \\
\hline
\end{tabular}

${ }^{2}$ Full bloom occurred 16 Apr. 2000. Treatments were applied at PF, $\mathrm{PF}+21, \mathrm{PF}+42$ days with a low pressure hand-wand sprayer.

${ }^{y}$ Concentrations: Apogee $(\mathrm{P}-\mathrm{Ca})=125 \mathrm{mg} \cdot \mathrm{L}^{-1}(\mathrm{P}-\mathrm{Ca}) ; \mathrm{CaCl}_{2}=0.454 \mathrm{~g} \cdot \mathrm{L}^{-1} ;\left(\mathrm{NH}_{4}\right)_{2} \mathrm{SO}_{4}=1.8 \mathrm{~g} \cdot \mathrm{L}^{-1}(\operatorname{trt} 12 \mathrm{only}), 0.454 \mathrm{~g} \cdot \mathrm{L}^{-1}$ (all other trts); Regulaid $=1.25 \mathrm{~mL} \cdot \mathrm{L}^{-1}$; Oil $=2.50 \mathrm{~mL} \cdot \mathrm{L}^{-1} ;$ Silwet L- $77=0.31 \mathrm{~mL} \cdot \mathrm{L}^{-1} \mathrm{NaCl}=0.454 \mathrm{~g} \cdot \mathrm{L}^{-1} ; \mathrm{KCl}=0.454 \mathrm{~g} \cdot \mathrm{L}^{-1} ; \mathrm{Li}-700=1.25 \mathrm{~mL} \cdot \mathrm{L}^{-1} ;$ Choice $=1.25 \mathrm{~mL} \cdot \mathrm{L}^{-1} ; \mathrm{Urea}=0.454 \mathrm{~g} \cdot \mathrm{L}^{-1} ; \mathrm{Captan}$ $=2.40 \mathrm{~g} \cdot \mathrm{L}^{-1} ;$ Ethephon $=270 \mathrm{mg} \cdot \mathrm{L}^{-1}$.

*Average length 10 longest scaffold shoots (26 June 00).

${ }^{\text {w} A v e r a g e ~ l e n g t h ~ a n d ~ w e i g h t ~ o f ~} 10$ longest scaffold shoots (5 Dec. 2000).

"Treated day after regular spray.

"Treated day of spray and allowed to dry before addition spray treatment was applied.

'Mean separation in columns by Duncan's New Multiple Range Test; $P \leq 0.05$. 


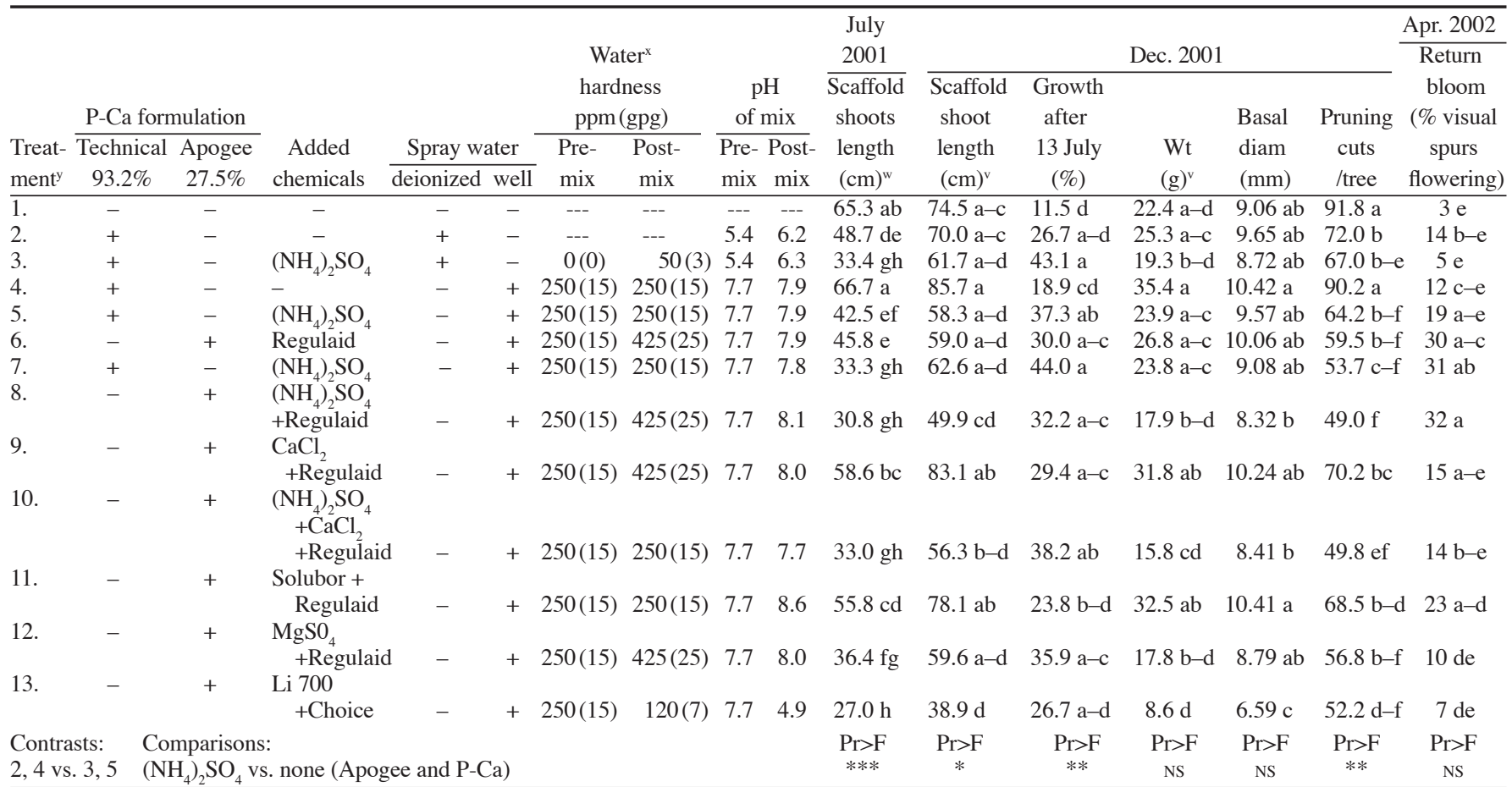

${ }^{2}$ Full bloom occurred 23 Apr. 2001. Treatments were applied at PF (30 Apr.), 23 d APF (23 May), and 52 d APF (21 June), with a low pressure hand-wand sprayer.

${ }^{y}$ Concentrations: $\mathrm{P}-\mathrm{Ca}=125 \mathrm{mg} \cdot \mathrm{L}^{-1} ;$ Apogee $=125 \mathrm{mg} \cdot \mathrm{L}^{-1}(\mathrm{P}-\mathrm{Ca}) ; \mathrm{CaCl}_{2}=2.4 \mathrm{~g} \cdot \mathrm{L}^{-1} ;\left(\mathrm{NH}_{4}\right) \mathrm{SO}_{4}=2.4 \mathrm{~g} \cdot \mathrm{L}^{-1}$ (trt 10 only), $0.454 \mathrm{~g} \cdot \mathrm{L}^{-1}$ (all other trts); Regulaid $=$ $1.25 \mathrm{ml} / \mathrm{L} ;$ Oil $=2.514$; Solubor $=1.20 \mathrm{~g} \cdot \mathrm{L}^{-1} ; \mathrm{MgSO}_{4}=6.0 \mathrm{~g} \cdot \mathrm{L}^{-1} ; \mathrm{Li}-700=1.25 \mathrm{~mL} \cdot \mathrm{L}^{-1} ;$ Choice $=2.50 \mathrm{~mL} \cdot \mathrm{L}^{-1}$.

${ }^{x}$ Water hardness was determined by dipping a Hach Sofchek Water Hardness Test Strip into each mix with either deionized or well water. Guidelines for water hardness are as follows: soft $=0-60 \mathrm{ppm}$; moderate $=61-120 \mathrm{ppm}$; hard $=121-180 \mathrm{ppm}$; very hard $=180 \mathrm{ppm} \&$ over; $(\mathrm{gpg})=$ grains per gallon: soft $=0-1$; slightly hard $=1-3.5$; moderately hard $=3.5-7.0$; hard $=7.0-10.5$; very hard $=\geq 10.5$.

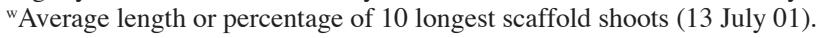

${ }^{v}$ Average length and weight of 10 longest scaffold shoots (4 Dec. 01).

ws, $, * *, * * *$ Nonsignificant or significant at $P \leq 0.05,0.01,0.001$, respectively.

days. In addition, Regulaid, $\left(\mathrm{NH}_{4}\right)_{2} \mathrm{SO}_{4}$, Choice, LI-700, $\mathrm{CaCl}_{2}$, Solubor (US Borax, Valencia, Calif.), and $\mathrm{MgSO}_{4}$ were applied in various combinations as potential adjuvants, or water conditioners in deionized water or "hard" well water [well water tested at $\approx 425$ $\mathrm{mg} \cdot \mathrm{L}^{-1}$ Calcium ion equivalents $(97 \% \mathrm{Ca}$ and $\mathrm{Mg}$ salts)] as indicated in Table 3. Ten of the longest scaffold shoots were tagged around the periphery of each tree and their lengths were measured on 13 July. During dormancy, these 10 scaffold shoots were removed and the basal diameters and shoot lengths were recorded. In addition, the nodes $/ \mathrm{cm}$ of the basal $40 \mathrm{~cm}$ and nodes $/ \mathrm{cm}$ of the upper $30 \mathrm{~cm}$ of shoots were recorded. Trees were pruned and the total length of the shoots longer than $30 \mathrm{~cm}$, the weight and time required to prune, the number of cuts, and the pruning weights $/ \mathrm{cm}^{2}$ TCSA per tree were recorded.

Expt.4. In 2001, 24 ten-year-old 'Empire'/ Mark trees (six blocks) were selected for four treatments (Table 4). P-Ca formulations [Apogee $27.5 \%$ a.i. P-Ca) or technical pro-hexadione-calcium (93.2\% a.i. P-CA)] were applied at $125 \mathrm{mg} \cdot \mathrm{L}^{-1} \mathrm{P}-\mathrm{Ca}$ in three applications to the same trees at $\mathrm{FB}+9, \mathrm{FB}+30$, and $\mathrm{FB}+64 \mathrm{~d}$. In addition Regulaid, $\left(\mathrm{NH}_{4}\right)_{2} \mathrm{SO}_{4}$, and/or Solubor, were applied in various combinations to determine if fruit cracking would occur (Table 4). On 19 July, 10 of the longest scaffold shoots were tagged around the periphery of each tree and their lengths were measured.

\section{Results and Discussion}

Inhibition of shoot growth by P-Ca was potentiated by $\left(\mathrm{NH}_{4}\right)_{2} \mathrm{SO}_{4}$ in experiments conducted in 1999, 2000, and 2001 on Fuji/ M.9 as measured by a suppression of scaffold shoot length, basal diameter, pruning weight (data not shown), and number of pruning cuts (Tables 1-3). The growth retardent diamozide suppressed shoot length in the year of application, but shoots grew longer the year after application and the basal diameter appeared to be increased and shoot weight unchanged in the year of application (Emerson and Byers, unpublished). Since the nature of diamozide growth suppression was not published, comparison of the shoot length, weight, and basal diameter cannot be compared to P-Ca. However, data reported here indicate that $\mathrm{P}$ $\mathrm{Ca}$ shoot suppression was not more vigorous in the subsequent season, as may have been with diamozide; thus from one season's applications, P-Ca truly suppressed tree vigor (Byers and Yoder, 1999).

Expt. 1. In 1999, the addition of $\left(\mathrm{NH}_{4}\right)_{2} \mathrm{SO}_{4}$ (treatments 4, 5, and 6 vs. 8, 11, and 15) to the Apogee sprays using hard well water (high in $\mathrm{Ca}$ and $\mathrm{Mg}$ ) improved efficacy by June 26 . The $10 \%$ P-Ca (BAS 125W) and the $27.5 \%$ $\mathrm{P}-\mathrm{Ca}$ (Apogee) formulations gave similar shoot growth suppression of scaffold shoot length by June 1999, when applied with Regulaid or Oil + Silwet L-77 (treatments 2 and 3 vs. 5 and 6, Table 1). The addition of $\mathrm{CaCl}_{2}$ to the Apogee $\left(\mathrm{P}-\mathrm{Ca}, 125 \mathrm{mg} \cdot \mathrm{L}^{-1}\right)$ formulation caused poorer scaffold shoot inhibition (treatments 4 , 5 , and 6 vs. 7, 10, and 14), but the addition of $\left(\mathrm{NH}_{4}\right)_{2} \mathrm{SO}_{4}$ restored the effectiveness of the mixture (treatments 7, 10, and 14, vs. 8, 11, and 15). When using "hard" well water, Apogee plus $\left(\mathrm{NH}_{4}\right)_{2} \mathrm{SO}_{4}$ promoted better activity (treatments 4,5 , and 6 vs. 8,11, and 15). The additives, $\left(\mathrm{NH}_{4}\right)_{2} \mathrm{SO}_{4}, \mathrm{CaCl}_{2}$, Regulaid, or Oil plus Silwet L-77, alone had no effect on scaffold shoot growth (1 vs. 16; 1 vs. 17). Apogee plus Silwet L-77 plus Oil provided additional shoot growth suppression when compared to Apogee plus Regulaid (treatments 2, 5, and 12 vs. 3,6 , and 13 ).

In 1999, scaffold shoot length grew little from June to December because tree carried a full crop of fruit. Although shoot lengths were significantly reduced for most all Apogee treatment $\mathrm{s}$, the basal diameters and the number of cuts required to prune the tree did not show the degree of growth suppression as did scaffold shoot length.

From a 10-fruit sample from each tree for treatments 1 and 11, Apogee $+\left(\mathrm{NH}_{4}\right)_{2} \mathrm{SO}_{4}+$ Regulaid did not affect fruit diameter, length/ diameter ratio, fruit firmness, SSC, starch fruit color, or fruit weight. Trees were thinned to about the same crop load $30 \mathrm{~d}$ after bloom (data not shown). No differences in fruit set were found.

Expt. 2. In 2000, the addition of $\left(\mathrm{NH}_{4}\right)_{2} \mathrm{SO}_{4}$ 


\section{Growth Regulators}

Table 4. Effect of P-Ca (3 applications) on shoot growth of 'Empire'/Mark applied in $2001^{\text {z }}$.

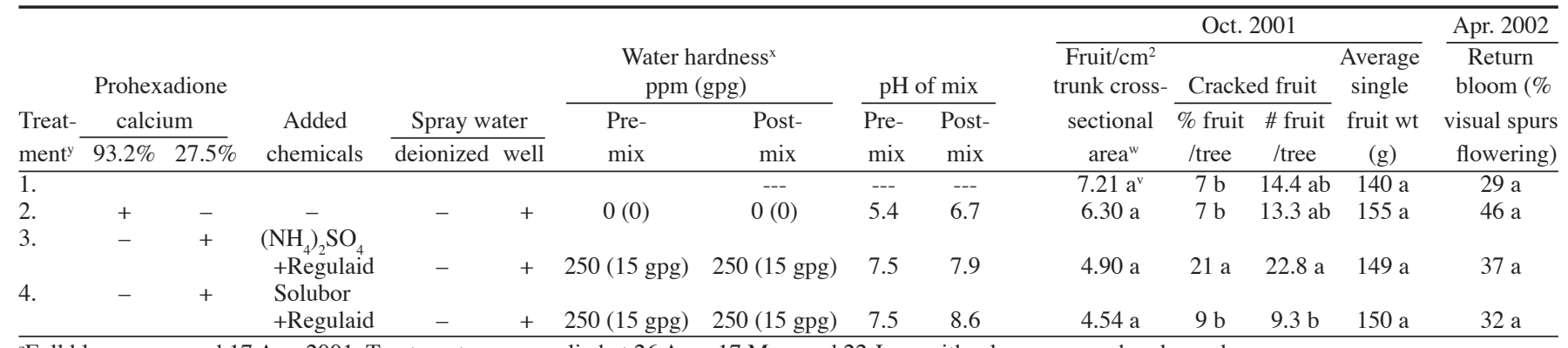

${ }^{\mathrm{z} F u l l}$ bloom occurred 17 Apr. 2001. Treatments were applied at 26 Apr., 17 May, and 22 June with a low pressure hand-wand sprayer.

${ }^{y}$ Concentrations $\left(\mathrm{mg} \cdot \mathrm{L}^{-1}\right)$ : PHCA $=125 \mathrm{mg} \cdot \mathrm{L}^{-1} ;$ Apogee $=125 \mathrm{mg} \cdot \mathrm{L}^{-1} ;\left(\mathrm{NH}_{4}\right)_{2} \mathrm{SO}_{4}=0.454 \mathrm{~g} \cdot \mathrm{L}^{-1} ; \mathrm{Regulaid}=1.25 \mathrm{~mL} \cdot \mathrm{L}^{-1} ; \mathrm{Solubor}=1.20 \mathrm{~g} \cdot \mathrm{L}-1$.

${ }^{x}$ Water hardness was determined by dipping a Hach Sofchek Water Hardness Test Strip into each mix with either deionized or well water. Guidelines for water hardness are as follows: soft $=0-60$ ppm; moderate $=61-120$ ppm; hard $=121-180$ ppm; very hard = 180 ppm \& over; $($ gpg $)=$ grains per gallon: soft $=0-1$; slightly hard $=1-3.5$; moderately hard $=3.5-7.0$; hard $=7.0-10.5$; very hard $=\geq 10.5$.

"Total fruit harvested.

'Mean separation within columns by Duncan's New Multiple Range Test $(P \leq 0.05)$

(treatment 2 vs. 3 ) or Choice + Li700 (treatment 2 vs. 7) to the Apogee sprays using hard well water (high in $\mathrm{Ca}$ and $\mathrm{Mg}$ ) improved efficacy on 26 June; but the addition of Urea, $\mathrm{NaCl}$, $\mathrm{KCl}$, or $\mathrm{CaCl}_{2}$ (treatment 2 vs. either treatments $4,9,10$, or 11 ) to Agogee was no better than Apogee alone (Table 2). The addition of Silwet $\mathrm{L}-77$ to $\left(\mathrm{NH}_{4}\right)_{2} \mathrm{SO}_{4}+$ Apogee further may not have improved efficacy (treatment $3 \mathrm{vs.} 8$ ). The addition of $\mathrm{CaCl}_{2}$ to the $\left(\mathrm{NH}_{4}\right)_{2} \mathrm{SO}_{4}+$ Apogee spray was not detrimental to shoot growth suppression if a high rate of $\left(\mathrm{NH}_{4}\right)_{2} \mathrm{SO}_{4}$ (equivalent to the $\mathrm{CaCl}_{2}$ rate $\mathrm{w} / \mathrm{w}$ ) was used (treatment 5 vs. 12). However, the addition of $\left(\mathrm{NH}_{4}\right)_{2} \mathrm{SO}_{4}$ at a low rate (equivalent to the Apogee rate $\mathrm{w} / \mathrm{w})$ to a tank mix of Apogee $+\left(\mathrm{NH}_{4}\right)_{2} \mathrm{SO}_{4}$ + Regulaid (treatment 5 vs. 13) efficacy was reduced. In addition, $\mathrm{CaCl}_{2}$ applied before or after Apogee + Regulaid did not reduce efficacy in this experiment (treatment s14 vs. 15, 14 vs. 16). Adjusting the $\mathrm{pH}$ of the Apogee + $\left(\mathrm{NH}_{4}\right)_{2} \mathrm{SO}_{4}+$ Regulaid spray to either $\mathrm{pH}=4$ or $\mathrm{pH}=9$ did not affect efficacy (treatment 17 vs. 5 , and 18 vs. 5). Tank mixing captan with Apogee $+\left(\mathrm{NH}_{4}\right)_{2} \mathrm{SO}_{4}+$ Regulaid did not cause foliage injury or affect efficacy (treatment 19 vs. 5). The addition of ethephon at $270 \mathrm{mg} \cdot \mathrm{L}^{-1}$ improved the growth suppression of Apogee $+\left(\mathrm{NH}_{4}\right)_{2} \mathrm{SO}_{4}+$ Regulaid (treatment 20 vs. 5). The additives Regulaid $+\mathrm{CaCl}_{2}+\left(\mathrm{NH}_{4}\right)_{2} \mathrm{SO}_{4}$ alone (treatment 21 vs. 1) did not cause shoot growth suppression or injury.

Data taken 5 Dec. 2000 on the average shoot length of the 10 longest shoots tagged on 26 June, showed no shoot inhibition by any of the Apogee sprays (Table 2); however, pruning cuts $/ \mathrm{cm}^{2}$ TCSA reflected similar growth suppression to shoot length data taken on 26 June. Those trees inhibited most by the treatments (based on the 10 tagged shoots on 26 June) also grew the most from 26 June to 5 Dec. 2000. Tagging the strongest shoots on the tree for measurements ignored less vigorous shoots that were reflected by the pruning cut data. Regrowth was probably related to no crop existing on the trees in this experiment. This was in contrast to Expt. 1, which had a full crop and did not have a significant regrowth.

Ethephon tank mixed with Apogee + $\left(\mathrm{NH}_{4}\right)_{2} \mathrm{SO}_{4}+$ Regulaid (treatment 20) promoted additional suppression of shoot growth.
Numerically shoot length, shoot diameter, and pruning weights were reduced more than any other treatment, and it was the only treatment to significantly increase return bloom.

Expt. 3. In 2001, prohexadione-calcium technical grade $(93.2 \%$ P-Ca) applied at 125 $\mathrm{mg} \cdot \mathrm{L}^{-1}$ to 'Fuji'/M.9 trees in three applications in deionized water reduced shoot growth by June 2001 (treatment 1 vs. 2), and the addition of $\left(\mathrm{NH}_{4}\right)_{2} \mathrm{SO}_{4}$ to P-CAfurther suppressed shoot growth (treatment 2 vs. 3) (Table 3). However, if P-CA was mixed in well water $\left(250 \mathrm{mg} \cdot \mathrm{L}^{-1}\right.$ hardness), P-CAdid not suppress shoot growth (treatment 2 vs. 4 ). Apogee (27.5\% a.i., $+72.5 \%$ $\left(\mathrm{NH}_{4}\right)_{2} \mathrm{SO}_{4}$ in the formulation) + Regulaid in well water was not as effective as simply adding more $\left(\mathrm{NH}_{4}\right)_{2} \mathrm{SO}_{4}$ (treatment 6 vs. 7); however, the Regulaid may have had some additional influence, but not statistically (treatment 7 vs. 8). If $\mathrm{CaCl}_{2}$ was added, the calcium completely inhibited the effect of the Apogee + Regulaid (treatment 6 vs. 9); but if $\left(\mathrm{NH}_{4}\right)_{2} \mathrm{SO}_{4}$ was added at the same rate $(\mathrm{w} / \mathrm{w})$, the effectiveness was completely restored (treatment $9 \mathrm{vs.} 10$ ). When Solubor was added to Apogee + Regulaid, effectiveness was compromised (treatment 6 vs. 11), but $\mathrm{MgSO}_{4}$ did not (treatment 6 vs. 12). Apogee + Li-700 + Choice, a commercial water conditioner, numerically was the most effective treatment (treatment 13). Choice, among other ingredients, has $\left(\mathrm{NH}_{4}\right)_{2} \mathrm{SO}_{4}$ in the formulation. In the dormant season, additional measurements were taken to document the amount of re-growth after 13 July. The amount of growth after 13 July was inversely related to amount of growth suppression from April to July. Nontreated trees that grew the most in the early season had the least amount of growth after 13 July. This was probably related to no crop existing on the trees in this experiment in contrast to Expt. 1 where a full crop may have greatly reduce re-growth after July.

Expt. 4. In 2001, Prohexadione-calcium [formulated as $27.5 \%$ P-Ca (Apogee) or the technical PHCA (93.2\% P-Ca] applied at $125 \mathrm{mg} \cdot \mathrm{L}^{-1}$ to 'Empire'/Mark trees in three applications at $\mathrm{FB}+9, \mathrm{FB}+30$, and $\mathrm{FB}+64 \mathrm{~d}$ in deionized water did not reduce tree growth (data not shown) due to heavily cropping trees. Only the combination of Apogee $+\left(\mathrm{NH}_{4}\right)_{2} \mathrm{SO}_{4}+$ Regulaid caused fruit cracking $(21 \%$; treatment
3) as compared to in the control (Table 4, treatment $1=7 \%$ ). Data from Expts. 1-3 suggest that this combination was more effective as a result of increased absorption or activation of $\mathrm{P}-\mathrm{Ca}$; thus, the cracking of 'Empire' may be related to a higher concentration of $\mathrm{P}-\mathrm{Ca}$ in the fruit or the tree.

\section{Conclusions}

The past three seasons' experiments support the benefit of adding additional $\left(\mathrm{NH}_{4}\right)_{2} \mathrm{SO}_{4}$ to the Apogee formulation [27.5\% P-Ca + 72.5\% $\left.\left(\mathrm{NH}_{4}\right)_{2} \mathrm{SO}_{4}\right]$ sprays, especially when using high calcium content well water. However, since $\left(\mathrm{NH}_{4}\right)_{2} \mathrm{SO}_{4}$ improved Apogee efficacy in deionized water, the additional benefit of $\left(\mathrm{NH}_{4}\right)_{2} \mathrm{SO}_{4}$ to all water sources may be important to maximizing efficacy. Ammonium sulfate is used to improve the uptake of many herbicides (Kapusta, 1996). Scanning electron micrographs of spray deposits of glyphosphate $+\mathrm{CaCl}_{2}$ showed an amorphous, thick, and noncrystalline deposit which may act to physically entrap glyphosate. When ammonium sulfate is added, leaf contact, thickness, and crystalline content was correlated with increasing toxicity in the leaf surface of wheat, sunflower, and kochia (Nalewaja et al., 1992). However, the function of $\left(\mathrm{NH}_{4}\right)_{2} \mathrm{SO}_{4}$ may be to provide a more efficient cation $\left(\mathrm{NH}_{4}^{+}\right.$vs. $\left.\mathrm{Ca}^{++}\right)$for the prohexadion anion. In addition, $\mathrm{NH}_{4}$ ions may increase the loading, transport, or both, of the P-Ca molecule to the site of GA inhibition by supplying a proton pump with sufficient inorganic energy (Harold, 1986; Sze et al., 1999). The adjustment of $\mathrm{pH}$ to 4 or to 9 (with $\mathrm{HCl}$ or $\mathrm{NaOH}$ ) did not result in a change in Apogee response (Expt. 2). However, during drying, the $\mathrm{pH}$ of the spray/plant surface was not known. A different result may have been obtained if $\mathrm{pH}$ buffered solutions were used. Mixing of $\mathrm{CaCl}_{2}$ with Apogee, in a beaker in the laboratory, did not produce a precipiate with well water or deionized water. For this reason, $\left(\mathrm{NH}_{4}\right)_{2} \mathrm{SO}_{4}$ probably affected $\mathrm{P}-\mathrm{Ca}$ absorption, translocation, or both, to the site of GA synthesis.

Hardness of water is frequently referred to as $\mathrm{Ca}^{++}$concentration equivalents, but also may be referred to by the precipitation 
of a soap with $\mathrm{Ca}^{++}$or other cations, such as $\mathrm{Mg}^{++}$. A water conditioner may change the precipitation properties of a calcium solution while not affecting the calcium concentration in the solution. When well-water "hardness" was increased by adding $\mathrm{CaCl}_{2}$ to the Apogee spray solution, the growth inhibition properties of $\mathrm{P}-\mathrm{Ca}$ was almost completely negated; however, $\mathrm{MgSO}_{4}$ did not have the same effect as $\mathrm{CaCl}_{2}$. The addition of an equal weight $\left(\mathrm{NH}_{4}\right)_{2} \mathrm{SO}_{4}$ restored the P-Ca activity, but adding either $\mathrm{NaCl}$ or $\mathrm{KCl}$ to the spray did not influence P-Ca activity even though both are used in cation exchange water softeners. $\left(\mathrm{NH}_{4}\right)_{2} \mathrm{SO}_{4}$ may have had a direct affect on uptake and/or activity of the P-Ca molecule by substituting the $\mathrm{NH}_{4}^{+}$for $\mathrm{Ca}^{++}$. The commercial water conditioner, Choice + LI-700 was also as effective as $\left(\mathrm{NH}_{4}\right)_{2} \mathrm{SO}_{4}+$ Regulaid for counteracting the effect of high calcium well water. Since Solubor also inhibited the activity of Apogee, additional studies on the nature of $\mathrm{P}-\mathrm{Ca}$ absorption, translocation, and/ or action could provide useful information for increasing efficacy of Apogee sprays.

The combination of ethephon + Apogee provided some additional suppression of shoot growth and increased flowering (Table 2). Since ethephon can cause fruit thinning during the period when $\mathrm{P}-\mathrm{Ca}$ is recommended, either low ethephon rates must be chosen in the $40 \mathrm{~d}$ AFB or it should be applied only during the ethephon insensitive fruit thinning period from $\approx 40$ to $70 \mathrm{~d}$ AFB (1 June-15 July).

Shoot growth suppression by $\mathrm{P}-\mathrm{Ca}$ in comparison to the control was considerably different between years (Expts. 1-3). Shoot inhibition measured 3 weeks after the last three sprays (in mid-June) appeared to be similar for the three experiments. However, in the latter half of the season, shoot growth was greatest in Expts. 2 and 3 when trees carried no fruit. The heavy crop load in Expt. 1 probably controlled shoot growth during the second half of the season. Soil moisture as affected by summer rain, humidity, and temperatures were different between the three seasons, but crop load was considered to be the primary factor leading to regrowth after the third P-Ca application.

\section{Literature Cited}

Byers, R.E. and K.S. Yoder. 1999. Prohexadioncalicum inhibits apple, but not peach, tree growth, but has little influence on apple fruit thinning or quality. HortScience 34:1205-1209.

Faust, M. (ed.). 1984. International workshop on controlling vigor in fruit trees. Acta Hort. 146.

Harold, F.M. 1986. The vital force: A study of bioenergetics. W.H. Freeman and Co.

Kapusta, G., 1996. A compendium of herbicide adjuvants. Dept. of Plant and Soil Sci., Southern Illinois Univ.

Looney, N.E. 1983. Growth regulator usage in apple and pear production, p. 27-40 In: L.G. Nickell (ed.). Plant growth regulating chemicals, Vol.
I. CRC Press, Boca Raton, Fla.

Luckwill, L. C. 1970. The control of growth and fruitfulness of apple trees, p. 237-254. In: L.C. Luckwill and C.V. Cutting (eds.). Physiology of tree crops. Academic, London.

Miller, S.S. 1988. Plant bioregulators in apple and pear culture. Hort. Rev. 10:309-401.

Nalewaja J.D., R. Matysiak, and T.P. Freeman. 1992. Spray droplet residual of glyphosate in various carriers. Weed Science 40:576-589.

Poapst, P.A., G.M. Ward, and W.R. Phillips. 1959. Maturation of McIntosh apples in relation to starch loss and abscission. Can. J. Plant Sci. 39:257-263.

Rademacher, W. 2000. Growth retardents: Effects on gibberellin biosynthesis and other metabolic pathways. Annu. Rev. Plant Physiol. Plant Mol. Biol. 51:501-31.

Raese and Drake. 2002. Calcium spray materials and fruit calcium concentrations influence apple quality. J. Amer. Pom. Soc. 53:126-143.

Sze H., L.Xuhang, and M.G. Palmgren. 1999.Energization of plant cell membranes by $\mathrm{H}^{+}$-Pumping ATPases: Regulation and biosynthesis. Plant Cell 11:677-689.

SAS Institute. 1985. SAS user's guide: Statistics. SAS Inst., Cary, N.C.

Williams, M.W. 1984. Use of bioregulators to control vegetative growth of fruit trees and improve fruit efficiency, p. 93-99. In: R.L. Orydn and F.R. Rittig (eds.). Bioregulators: Chemistry and uses. Amer. Chem. Soc. Wash., D.C.

Yoder, K.S., S.S. Miller, and R.E. Byers. 1999. Suppression of fireblight in apple shoots by prohexadion-calicum (BAS125W) following experimental and natural inoculation. HortScience 34:1202-1204. 